\title{
INACTIVATION OF ENVELOPED VIRUSES BY A SILVER-THIOSULFATE COMPLEX
}

\author{
Hiroaki Oka ${ }^{1}$, Toshikazu Tomioka', Katsumi Tomita', \\ Atsushi Nishino ${ }^{1}$ and Shigeharu Ueda ${ }^{2}$ \\ ${ }^{1}$ Matsushita Electric Industrial Co., Ltd., 3-1-1, Yagumo-Nakamachi, Moriguchi, Osaka 570, Japan \\ 2 Department of Neurovirology, Research Institute for Microbial Diseases, Osaka University, \\ Yamada-oka, Osaka 565, Japan
}

Amenitop ${ }^{\mathrm{TM}}$ was developed by Matsushita Electric Industrial Co., Ltd. It is an inorganic material originally developed to prevent bacterial contamination on the surface of appliances such as telephone and facsimile machines, and consists of silica gel microspheres containing silverthiosulfate complex (AT-1). It has a coating layer of tetraethoxysilanet that enables gradual release of AT-1 on the surface. AT-1 showed bactericidal effect on various kinds of bacteria such as $E$. coli, S. aureus, methicillin resistant $S$. aureus (MRSA). So, we examined antiviral effect of AT-1 on several kinds of viruses such as human immunodeficiency virus-1 (HIV-1, JMH-1), herpes simplex virus type-2 (HSV-2, G strain), measles virus (MV, Nagahata strain) and polio virus type1 (PV, Sabin vaccine type 1$)$.

AT-1 was extracted into phosphate buffered saline (PBS) from Amenitop ${ }^{\mathrm{TM}}$ at $37 \mathrm{C}$. for $30 \mathrm{~min}$ to obtain pure AT-1. The extracted AT-1 was stored at $-80 \mathrm{C}$ until use. HIV-1 was propagated in MT-4 cells. HSV-2, MV and PV were propagated in Vero cells. Stock viruses were prepared from the culture supernatant fluids by low speed centrifugation and stored at $-80 \mathrm{C}$ until use.

An aliquot of $500 \mu$ l of the stock virus and AT-1 was mixed and incubated at $37 \mathrm{C}, 22 \mathrm{C}$ or $4 \mathrm{C}$ for a given time. AT-1 was removed from the mixture by centrifugation using Centrisart (Sartorius Co. Ltd., cut off M.W. 20000) after the incubation. Then, the mixture was supplemented with $1 \mathrm{ml}$ of PBS. Virus infectivity was measured using microplates by observing cytopathic effect.

AT-1 reduced infectivity of HIV-1103 $\mathrm{TCID} 50 / \mathrm{ml}$ in $30 \mathrm{~min}$ at $37 \mathrm{C}$. The inactivating effect of AT-1 on HIV-1 was temperature-dependent and it failed to inactivate HIV-1 at $4 \mathrm{C}$. Further, we examined the inhibiting effect of AT-1 on HIV-1 multiplication. Twelve nM of AT-1 in the culture medium inhibited multiplication of HIV-1 during 4 to 7 days culture after infection at multiplicity of infection 0.001 without any cytotoxicity.

Likewise, AT-1 inactivated MV10 ${ }^{4} \mathrm{TCID}_{50} / \mathrm{ml}$ at $37 \mathrm{C}$ in $30 \mathrm{~min}$. Temperature dependency of the inactivating effect of AT-1 was the same as in the case of HIV-1 and it failed to inactivate MV at 4 $C$ even after 3 hours incubation. Inactivation of HSV-2 and PV with AT-1 was similarly examined. AT-1 inactivated HSV-2 $10^{6} \mathrm{TCID}_{50} / \mathrm{ml}$. However, PV did not show any reduction in infectivity. The results suggest membrane-destroying effect of AT-1. 